\title{
A review of high-level robot functionality for elderly
}

care

\author{
Nico Sun ${ }^{1}$, Erfu Yang ${ }^{1}$, Jonathan Corney ${ }^{1}$, Yi Chen $^{2}$ and Zeli Ma \\ 1. Department of Design, Manufacture and Engineering \\ Management \\ University of Strathclyde, Glasgow \\ GlasgowG1 1XJ, U.K. \\ \{nico.sun, erfu.yang, jonathan.corney\}@strath.ac.uk \\ 2. Rofut (Shanghai) Technology Co.,Ltd \\ Shanghai 201800, China \\ \{leo.chen, macius.ma\}@rofut.com
}

\begin{abstract}
This paper presents an evidence-based overview of the functionality that robotic care systems should provide. The results identify a number of key characteristics that range from existing commercial products to research prototypes. For example, social care needs voice assistance that already exists in the form of smart speakers. Such systems provide an opportunity for entertainment and the ability to stay in contact with caregivers, friends and family. Consequently, a good speech recognition and ability to perform conversations were highly valued by elderly users. In contrast, care robots providing physical assistance still have not left the prototype phase and generally, do not have enough skills to be considered useful in the home. The results highlight the fact that the most common difficulties the elderly experience have not been solved and should be focused on in the future. The perception of usefulness and integration into the existing home of an elderly person are the main barriers to a robot being accepted as a part of the household. One of the paper's conclusions is that an ecosystem open to independent developers could greatly increase the robotic skillset and the chance that it can perform a useful task for its user.
\end{abstract}

Keywords-healthcare robotics, elderly care needs, care robot acceptance, robot skills

\section{INTRODUCTION}

The number of people aged 65 and older is projected to grow from an estimated 524 million in 2010 to nearly 1.5 billion in 2050, with most of the increase in developing countries [1].

This increase is not only in absolute numbers, but also relative to the total population of developing countries. Flandorfer estimates that in 2050 the old age dependency ratio (population outside of the working age) in the US will be $34 \%$, in the EU $49 \%$ and in Japan $76 \%$ [2]. The relative increase of older people is a more serious concern because fewer people of working age are able to care for the elderly, bringing economic and personal health problems.

According to the charity AgeUK [3], most of the current care is informal by life partners, family and friends. Currently, the value of informal care (£132 billion) exceeds the total NHS budget ( $£ 117.2$ billion in $2015 / 16)$. From the development of home care vs nursing/day-care, this relationship is not expected to change. Further many carers are elderly themselves with around 1 in 5 people aged 65 and over providing care. The high proportion of informal care also creates an information gap. According to [4] most care for the elderly is provided by informal carers such as spouses, children and other relatives. These secondary users live very close to the primary user of the care robot, might want to use the robot as well and their interaction with the primary user should not be hindered by the robotic carer.

In order to reliably design care systems for millions of people all requiring individual care, it is essential to correctly identify what living difficulty occurs how often, at what age it becomes common and how the current care situation for the issue is. For a robotic care system to be accepted into the elderly home designers should also know what drives people to welcome robots into their life.

The rest of the paper is structured as follows: the next section of this review summarizes the elderly care needs, followed by the care skills of existing care systems. The $4^{\text {th }}$ section of the review presents elderly acceptance of robotic care systems and their wishes in terms of physical appearance and skill set. Finally, the review discusses 
options for the future development of care robots to meet all of these requirements.

This review concentrates on papers published in the last 5 years and prioritizes the most recent published papers due to the rapid development of home care systems and the demographic progression.

\section{OVERVIEW OF ELDERLY CARE NEEDS}

Because of the high proportion of informal care, there is a lack of robust statistics about the needs of all elderly and the information that exists is usually not connected to robotic healthcare research, but to economic analysis, government planning or general health statistics.

Around $30 \%$ of the elderly live alone and $12 \%$ claim to be persistently / chronically lonely. The risk of premature death rises with loneliness up to a quarter [5]. Other studies estimated it to be as harmful as smoking 15 cigarettes a day [6]. Affected persons are also twice as likely to develop Alzheimer's than people with a low degree of loneliness.

Physical care needs are known as difficulties with "activity of daily living" (ADLs) such as bathing, getting in or out of bed, dressing, eating, use of the toilet or walking across the room. For each of the mentioned ADLs around $50 \%$ receive the help that meets their needs, $30 \%$ receive help, that doesn't meet their needs and $15-$ $20 \%$ don't receive any help at all.

Very common difficulties with ADLs are taking one flight of stairs $(22 \%)$, working around the house or garden $(18 \%)$, dressing (16\%), shopping groceries and bathing $(13 \%)$. Less common are eating $(3 \%)$, using the toilet (4\%) and walking 100 yards (4-5\%).

Elderly care doesn't typically involve treatment, but it can limit the progression of some diseases. For example, dementia becomes increasingly common over the age of 80 and affects up to $30 \%$ of females at the age of 95 . Studies have shown that a mentally more active life decreases the risk of cognitive impairment [7]. Like a human carer, a robot can interact over visual, audible \& physical methods actively stimulating more than one sense or keep communication possible in case one of the interactions becomes difficult for a person.

\section{Detailed ADL charts can be found in the Appendix}

\section{EXISTING CARE SYSTEMS}

\section{A. Smart Home Assistance}

Smart homes are not specifically designed to support the elderly, but home occupants in general and make the use of the Internet of things (IoT). Their common aspects include automatic lights, curtains, fridge ordering new food, etc.

All devices are usually connected to the main hub, in recent years several technology companies have developed general voice assistants which work as hubs (in the format of a loudspeaker) e.g. Amazon Alexa (Echo), Google Home, Cortana speaker. The users typically interact with these devices verbally, demanding a good voice recognition and "intelligence" to process their enquiries. Alexa, Siri, and Cortana are personified each having a specific name, gender and personality, hence encouraging the users to anthropomorphize the device.

Current functions include control of other devices (IoT) in the home, learn new skills or help the user directly. In pilot programs elderly could use the devices to call friends/family, make plans, web searches or request weather updates. While not providing any physical support these smart devices help elderlies to stay more connected to the outside world, manage a social life and organizing their life [8].

Based on 587 detailed Amazon.com reviews (December 2016) about the Assistant Alexa and not the hardware or delivery, a study found a correlation between satisfaction and personification of the device. And households with other family members using the device were more likely to personify the device than a single user. The assistant was also twice as often used for Entertainment (79\%) as for providing Information (39\%) or behaving as an assistant (33.4\%). Only 5.5\% of the reviewers mentioned the use as a companion and $7.2 \%$ mentioned the use of a friend [9].

\section{B. Smart Wheelchair}

Smart or intelligent wheelchairs were developed from powered (electric) wheelchairs. Powered wheelchairs, in turn, were developed for motoric disabled people who can't turn the wheels themselves or don't have a carer to push the wheelchair. According to [10] 15\% of wheelchair users require a powered wheelchair. Of the powered wheelchair users, $10 \%$ are unable to use their wheelchair for their ADLs [10].

Reasons for not meeting ADLs include: controlling the PW's joystick, avoiding obstacles, manoeuvring inside a constrained space, manoeuvring backwards and going through small doorways [10].

For this group, smart wheelchairs offer a solution to some of the mentioned problems. Autonomous movement and a new user interaction either by the graphical user interface (GUI), voice control or brain-computer interface (BCI) can solve the joystick issue and avoid obstacles. Manoeuvring in constrained spaces and fitting through small doorways, on the other hand, can become harder because a smart wheelchair needs sensors around the wheelchair itself for navigation, which increase its size. A computer providing the processing power for navigation and user interaction further adds weight and size to the wheelchair.

\section{Service Robots}

In the home environment service robots support people with physical daily living tasks such as preparing a drink, eating or bringing objects. The general goal for service robots is to perform household tasks that have become too difficult for the user or are perceived as too time-consuming or unpleasant. With a reasonable speech recognition and AI, service robots could as well become the main interaction point for a smart home and control other assistive devices. 
Some service robots have very specific designs focusing on one particular activity such as vacuum cleaning robots or RIBA (Robot for Interactive Body Assistance) that helps people to get out of bed and move them to another location. While robot vacuum cleaners have been made commercially available other specific robots have been so far been predominantly a technology demonstration. With a cost of $£ 57,000$, the RIBA II is comparable to a luxury car and more than double the median UK household income $(£ 26,300 ; 2016)$ [11].

Multi-function service robots have been predominantly prototypes too and multi-year projects by major research organisations such as the Care-O-bot project by Fraunhofer IPA. The first generations (Care-Obot I (1998) consisting of a touchscreen interface could navigate people in public environments. The main goal was to integrate the mobility skills. The Care-O-bot II (2002) concentrated on mobility skills. Care-O-bot I on navigation in a room and Care-O-bot II on a manipulator's arm to hold and operate basic household items and a laser scanner to recognize different objects. For the robot, Fraunhofer IPA also developed a "Robotics Toolbox", a software library which contains individual packages for robot control, e.g. autonomous navigation, obstacle avoidance, sensor drivers and actuator drivers. The individual packages can be used for different systems [12].

Care-O-bot 3 (2008) was designed as a butler, but not as a humanoid to avoid unrealistic expectations by users and increase the acceptance. It has been used in care assistance trials to e.g. bringing a drink to the user, follow a user, detect objects, and suggest activities [13].

The fourth generation of the Care-O-bot project, CareO-bot 4 (2015) is more flexible, supporting a modular design allowing users to configure it to their needs and wishes. Apart from homecare/helper, the robot can also operate in public spaces, like museums, airports, stores and security applications.

The most common skills developed for service robots are medication management, monitoring, and emergency help, bringing objects or feeding. Other developments, shared with social robots are entertainment, companionship and communication [14].

Due to their size and autonomous mobility service robots have a 'presence' in the home and can provide some form of companionship like a pet. The ACCOMPANY design accomplished to some users to feel an attachment towards it [15]. This would address the social care challenge of the loneliness of the elderly better than a smart hub in the form of a loudspeaker or a smart wheelchair where the user is part of the device (sitting on it), always moving with the user and being surrounded by it.

\section{ACCEPTANCE OF CARE ROBOTS BY THE ELDERLY}

Due to the limited availability of robot carers very few studies investigated the acceptance of different robots. Indeed most acceptance studies just looked at the acceptance of one prototype which was developed with specified goals. During tests users typically were interested and enthusiastic about the robot interaction, but most studies were not long-term studies. Simply the act of doing something new in a routine lifestyle can provide excitement and positive responses.

In 2015 a German case study in senior apartments found that real environments contained special challenges for robot navigation including difficult lighting conditions and various immobile obstacles that are difficult to detect (e.g. low jutting edges or glass-topped tables). The test users, which were already familiar with the robot's abilities and interactions rated them high in co-experience, indicating that it has a "high potential to be accepted as a companion and health supporter in everyday life of older people." [14]

A great desire was expressed for more functions \& better speech recognition. One user specifically wished the robot could support her husband while she has to "leave the house or to support him with exercising. She also would be delighted to use the robot as a partner for gaming." Due to the complexity of the apartments used during tests all apartments were mapped before. It was not investigated how the robot performed in an unknown environment (unmapped apartment and users without previous experience of the robot). Further evaluation would need a long-term test, determining if over a longterm the tendency to use the robot increase or decreases. Reason for more use can be increased trust and reason for less use can be decreasing interest over time.

In a TEDxTalk, Jenay Beer presented findings that elderly preferred a robot carer over a human carer in some instances. The participants answered the questionnaire after seeing a robot that could move some objects. The participants preferred the robot at cleaning the house, locate and retrieve objects which are positioned very low or high and to lift and move heavy objects. A human was preferred for personal care, e.g. bathing, shaving or preparing a meal [16].

Similar user acceptance was found by the EU robot project HOBBIT. The users highly appreciated robot skills like picking up objects from the floor and transporting them. Other valued skills were emergency recognition, a fitness program, and giving reminders [17]. Areas needing most improvement were voice control (speech recognition), gesture control and general responsiveness of the robot which seemed to be too slow.

Residents in a retirement village preferred a robot of $1.25 \mathrm{~m}$ height with wheels and skills to detect falls, control appliances, lift objects, cleaning, medication reminding [18]. They wished that the size should not be too intrusive and overpowering for older adults. The height preference had a widespread with $81 \%$ of participants choosing between $1.0 \mathrm{~m}$ and $1.5 \mathrm{~m}$. No clear preference was found for the robots gender, age, general shape, colour, humanlike appearance and voice.

Further insight into the acceptance of home robots into the life of the elderly is presented in the thesis by Susanne Frennert [19]. Most acceptance and integration of robots into their lives were for robots that supported everyday practices such as vacuum cleaning, rest, social activities and hobbies. Robots that focused on treating the user's 
health decline were less accepted. After the test, most participants were inclined to purchase their robot (eHealth system, assistive robot or vacuum cleaning robot) stating either that they don't need it or they expect better systems in the following years. Even the most accepted type (robot vacuum cleaner), which most users wanted to keep after the test wasn't conceived as a valuable purchase. This contradiction might be explained with Gerotranscedence, suggesting that older people are averse to owning new objects because they aim to dissolve their home when they die.

Smart wheelchairs can experience additional acceptance difficulties by elderly who suffer from incontinence or pressure sores. Not only would a smart wheelchair need to be proofed against incontinence, but it would also require additional cleaning maintenance. Both diseases generate negative feelings while directly using it. For couples, a smart wheelchair would only be able to assist one user directly as it is tied to one and can't move independently. A graphical user interface and navigation sensors in front of the user can also block the forward vision and hinder communication with other people.

\section{DISCUSSION \& FUTURE CARE ROBOT SKILL DEVELOPMENT}

Currently, the lack of commercial availability of care robots limits studies on long-term usage and acceptance by independent organisations. The few commercially available home care robots that could be used in long-term studies are the zoomorphic pet robots, voice assistance systems and robotic vacuum cleaners.

Existing research and prototypes of service robots for physical care concentrated on combining all parts of a robot including speech recognition, computer vision, navigation, actuator control and central coordination of all sub-systems. This could be seen as the first system integration challenge. The second integration challenge is into a person's home and to perform useful tasks. Current robotic care skills for ADLs are limited to the simpler tasks like eating, managing money and taking medication, which are the less common difficulties as well. Most elderly also already receive support for these difficulties.

The most common difficulty of daily living and least received help; climbing one flight of stairs is still largely unexplored. Similarly all tasks around the house and garden or help with dressing are still in need of a robot skill.

The robot acceptance research shows that most acceptance of a robot in the home environment is when it performs daily activities or provides entertainment. Even elderly people who would most need an assistant due to health issues view robots focusing mainly on their health decline as not desirable. They do not just accept any technology developed for them but decide on their own which to reject and which to use [19].

In real-world prototype testing, it was found that real senior apartments were much more crowded with obstacles and have narrower passages than expected or in trials in a designed apartment [20].
Obstacles in a home can be of different types. Permanent obstacles ( e.g. a cupboard, a sofa), semipermanent obstacles e.g. a large plant in a pot is usually not moved but might be moved due to rearranging the furniture or to make room to transport another large object. Lastly movable obstacles like a cushion lying on the floor or an open cabinet door blocking a narrow corridor. This problem is known as navigation among movable obstacles (NAMO) but hasn't been tested in realworld apartments yet. If a care robot would not be able to move because of a simple issue for a human like a cushion on the floor then acceptance would not be very high for the robot.

A simple manipulation of movable objects was performed in [21]. By AR tagging all objects as either movable or non-movable an overhead camera identified the location and category of the object. The robot then simply grabbed the movable obstacles with an arm and transported them to an empty location.

A more advanced NAMO skillset for the home was demonstrated in [22][23]. A small two-wheeled robot with two arms could manipulate a fixed set of rigid-body furniture to get to a goal. By using physics-based reinforcement learning (PBRL) the robot could pre-plan the manipulation of movable obstacles and adapt if they didn't behave as expected(locked wheel on the table). For a general service robot, this skill set would need to be enhanced for objects with unknown physics and objects which are not AR tagged for identification.

The NAMO navigation skills could also be used for complex housekeeping skills such as vacuum cleaning. Instead of vacuum around a bin and leaving a space unworked. The robot can temporarily move the object and clean the whole floor without gaps. Another possible application is pushing a cart or wheelchair. Instead of moving an obstacle out of the way a service robot could move an object (cart) towards a target. It could replace a smart wheelchair while avoiding several of the previously mentioned drawbacks.

The unwillingness of the elderly to purchase a robot due to gerotranscedence could be circumvented by marketing care robots as a service, paid over time, like a human carer instead of a complete purchase of a product. Apart from the effect of avoiding high purchase costs and easier return, paying for a robot as a service could also increase the likelihood of the robot being accepted as a companion because companions are not owned by other people. Future studies have to find out if acceptance of home robots is higher as a service.

The biggest advantage of a service model is probably that it can be used to create a robotic ecosystem. The current lack of care robot skills and its resulting impression not being useful enough has happened before in the tech industry. Windows Phone entered the smartphone market late, had a very low market share and very few developers wanted to develop apps for it. At the same time due to the app shortage, most people didn't want a Windows phone. The same lack of an ecosystem applies to voice assistants. Siri was the first voice assistant widely available since 2011 shipped with every iPhone, 
but in 2018 the voice assistants of smart speakers like Amazon Echo and Google Home are higher rated due to their service integration and open skills development from independent developers [25][26]. While Siri remained a product itself with a fixed set of skills other voice assistants created an ecosystem of voice assistant skills including control of smart home appliances.

If a care robot provides access to a robotic skills ecosystem where independent developers can receive a share of the revenue it encourages the development of more skills than any organisation could do by itself.

First steps towards a robotic ecosystem have been undertaken with universal control e.g. Robot Operating System (ROS) but without any commercial availability of service robots and currently, no business model to share revenue development is limited to a few funded research organisation and not thousands of developers like the smartphone market or the voice assistant market.

Of course, it must be said that currently there isn't a legal framework for such a model. In case the robot damages something it is unknown who would be responsible and has to pay for the damage. It could be the user, the skills developer or the robot manufacturer.

\section{CONCLUSION}

Many different robots have been developed in order to improve the quality of life of the elderly, but only a few have moved beyond the prototype stage and been made commercially available. At present most successful are social robots for the social care needs in form of general home assistance (smart speakers) allowing users to receive news updates, entertainment and stay in contact with friends and family.

In trials independently moving home care robots have been perceived to operate safely and are not feared by the elderly. Some daily activities are even preferred to be done by a robot over a human carer. However, currently, the limited skills of service robots and their high cost inhibit their acceptance and commercial availability.

The future challenge will be to integrate a home care robot into a person's home and life. More robot skills help the acceptance of a robot and contribute towards the feeling of being a useful addition to the home. Importantly the skills should be focused on maintaining everyday practices instead of emphasising the users' decline. In a home, the care robot must be able to move through a cluttered environment and handle obstacles in his path.

Creating an ecosystem for a product is a proven concept towards greater acceptance by its users. This happened previously with voice assistants and smartphone operating systems. In an open ecosystem, independent developers can create far more skills than any single organisation and increase the likelihood that a device can do something useful for a user.

\section{REFERENCES}

[1] Ruijiao Li;Bowen Lu, "Cognitive assisted living ambient system: a survey," Digit. Commun. Networks, vol. 1 , no. 4, pp. 229-252, Nov. 2015.
[2] P. Flandorfer, "Population Ageing and Socially Assistive Robots for Elderly Persons: The Importance of Sociodemographic Factors for User Acceptance," Int. J. Popul. Res., vol. 2012, pp. 1-13, 2012.

[3] "Briefing: Health and Care of Older People in England 2017, ," 2017.

[4] J. Roberts, H. Young, K. Andrew, A. McAlpine, and J. Hogg, "The needs of carers who push wheelchairs," J. Integr. Care, vol. 20, no. 1, pp. 23-34, 2012.

[5] AgeUK, "Later Life in the United Kingdom," 2017.

[6] J. Holt-Lunstad, T. B. Smith, and J. B. Layton, "Social Relationships and Mortality Risk: A Meta-analytic Review," PLoS Med., vol. 7, no. 7, p. e1000316, Jul. 2010.

[7] Alzheimer's Research UK, "Mental activity linked to slower cognitive decline | Alzheimer's Research UK," 2012. [Online]. Available: https://www.alzheimersresearchuk.org/mental-activity-

linked-to-slower-cognitive-decline-2/. [Accessed: 11Nov-2017].

[8] MIT TECHNOLOGY REVIEW, "MIT TECHNOLOGY REVIEW The Octogenarians Who Love Amazon 's Alexa,” vol. 120, no. 5, 2017.

[9] A. Purington, J. G. Taft, S. Sannon, N. N. Bazarova, and S. H. Taylor, "Alexa is my new BFF': Social Roles, User Satisfaction, and Personification of the Amazon Echo," 2017.

[10] C. Torkia et al., "Power wheelchair driving challenges in the community: a users' perspective.," Disabil. Rehabil. Assist. Technol., vol. 10, no. 3, pp. $211-$ 5, May 2015.

[11] Office for National Statistics, "Household disposable income and inequality in the UK: financial year ending 2016," 2017. [Online]. Available: https://www.ons.gov.uk/peoplepopulationandcommunity/ personalandhouseholdfinances/incomeandwealth/bulletins /householddisposableincomeandinequality/financialyearen ding2016\#median-household-disposable-income-1000higher-than-pre-downturn-level.

[12] M. Hans, B. Graf, and R. D. Schraft, "Robotic Home Assistant Care-O-bot: Past - Present - Future," 2002.

[13] FraunhoferIPA, "ACCOMPANY - Integrated robot technologies for supporting elderly people in their homes," 2014

[14] N. Doering et al., "Robotic Companions for Older People: A Case Study in the Wild 147 a," Annu. Rev. Cybertherapy Telemed., 2015.

[15] T. Sorell and H. Draper, "Robot carers, ethics, and older people," Ethics Inf. Technol., vol. 16, no. 3, pp. 183-195, Sep. 2014.

[16] TED, "Jenay Beer - Meet Your Grandparents' Assistant: The Domesticated Robot," TEDx Talks, 2012.

[17] E. Martinez-Martin and A. P. del Pobil, "Personal Robot Assistants for Elderly Care: An Overview," Springer, Cham, 2018, pp. 77-91.

[18] E. Broadbent, R. Tamagawa, N. Kerse, B. Knock, A. Patience, and B. MacDonald, "Retirement home staff and residents' preferences for healthcare robots," in RO-MAN 2009 - The 18th IEEE International Symposium on Robot and Human Interactive Communication, 2009, pp. 645650 .

[19] S. Frennert, "Older People Meet Robots Three Case Studies on the Domestication of Robots in Everyday Life," Lund University, 2016.

[20] H.-M. Gross et al., "Robot companion for domestic health assistance: Implementation, test and case study under everyday conditions in private apartments," in 2015 IEEE/RSJ International Conference on Intelligent Robots and Systems (IROS), 2015, pp. 5992-5999.

[21] E. Mueggler, M. Faessler, F. Fontana, and D. Scaramuzza, "Aerial-guided navigation of a ground robot 
among movable obstacles," 12th IEEE Int. Symp. Safety, Secur. Rescue Robot. SSRR 2014 - Symp. Proc., 2014.

[22] J. Scholz, N. Jindal, M. Levihn, C. L. Isbell, and H. I. Christensen, "Navigation Among Movable Obstacles with learned dynamic constraints," in 2016 IEEE/RSJ International Conference on Intelligent Robots and Systems (IROS), 2016, pp. 3706-3713.

[23] J. K. Scholz, "Physics-Based Reinforcement Learning for Autonomous Manipulation Physics-Based Reinforcement Learning for," no. December, 2015.
[24] M. Levihn, J. Scholz, and M. Stilman, "Hierarchical Decision Theoretic Planning for Navigation Among Movable Obstacles," Springer, Berlin, Heidelberg, 2013, pp. 19-35.

[25] "Amazon vs. Google: The Battle for Smart Speaker Market Share." [Online]. Available: http:/www.visualcapitalist.com/smart-speaker-marketshare/.

[26] “'I'm Not Sure I Understand'-How Apple's Siri Lost Her Mojo."

\section{APPENDIX}

Detailed elderly care chart by AgeUK showing the percentage of people who experience a specific difficulty on the x-axis and the percentage of people who receive help for the difficulty on the y-axis.

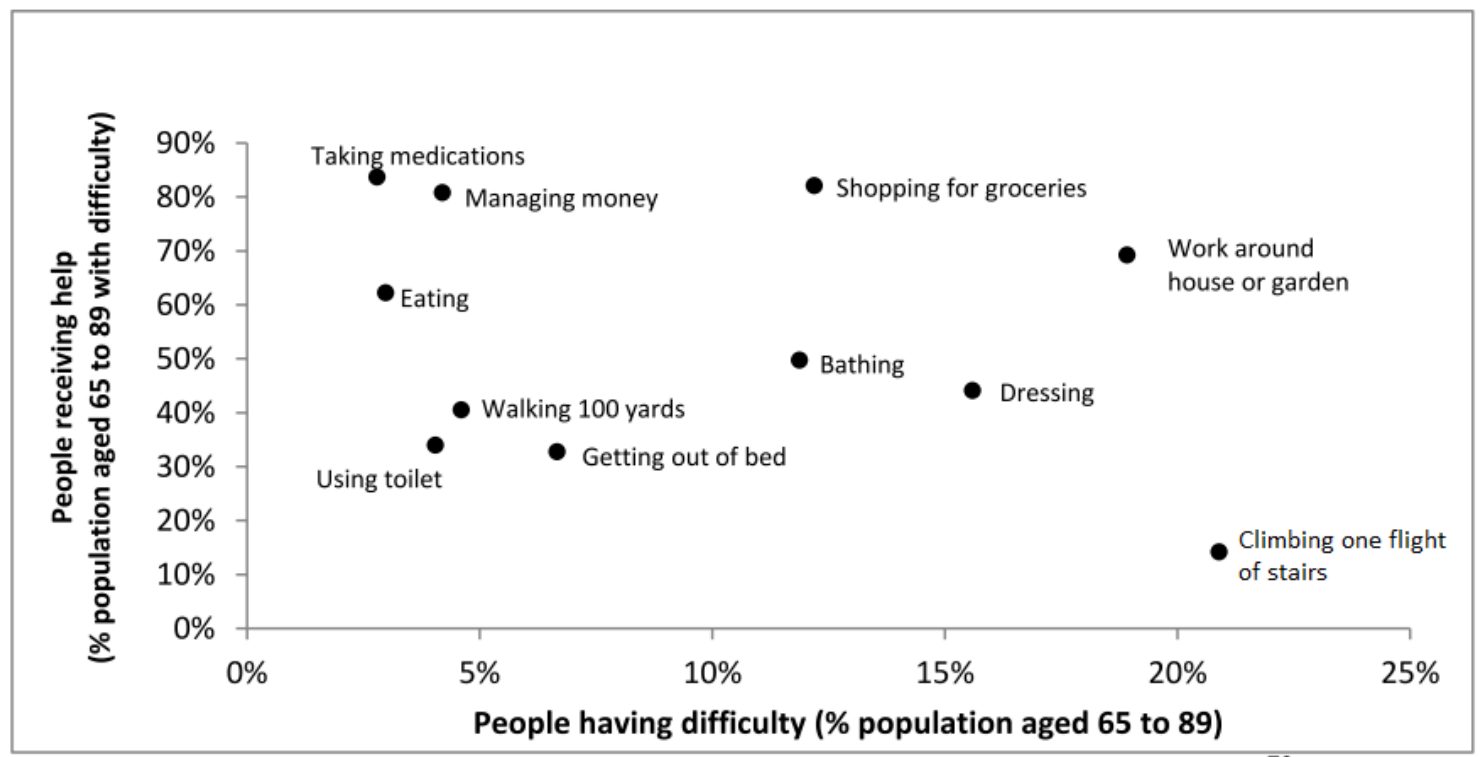

Source: Age UK analysis of the English Longitudinal Study of Ageing (2014)

Breakdown of individual difficulties and whether the help they receive meets their needs or if help is received at all.

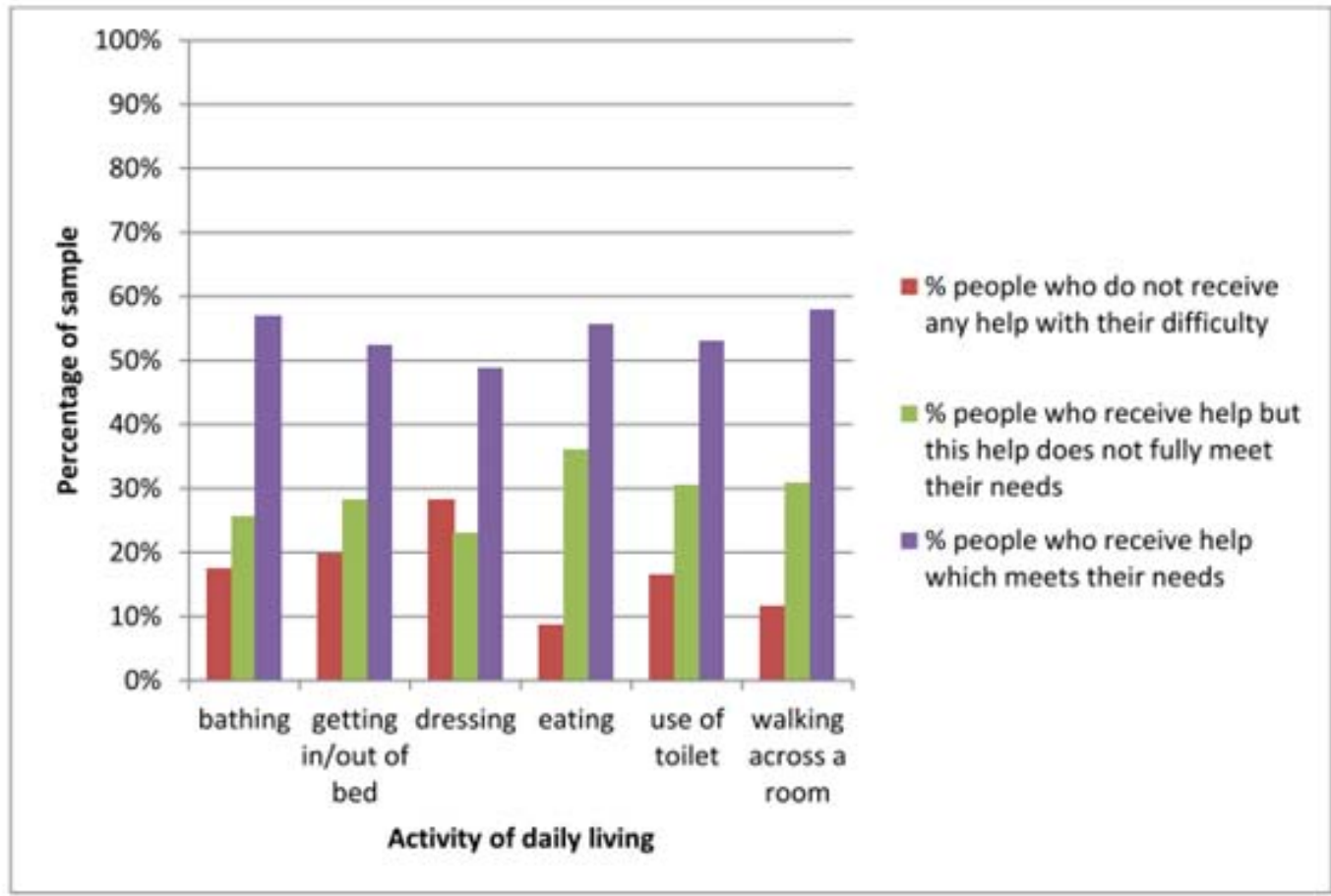

Source: English Longitudinal Study of Ageing (2016) 